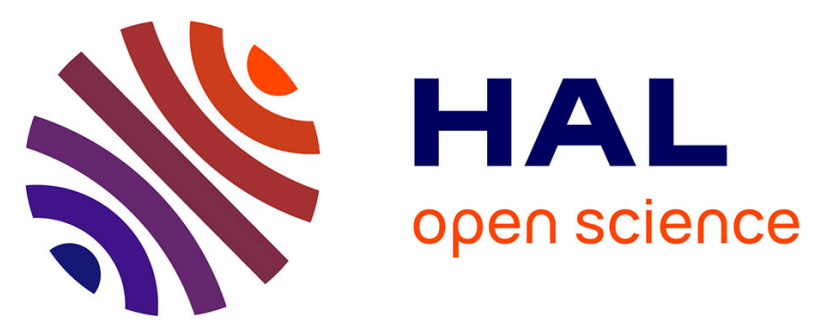

\title{
Performance impact of packet multiplexing on massive multiplayer online games
}

Dammak Marwa, Yassine Boujelben, Noura Sellami, Iryna Andriyanova

\section{To cite this version:}

Dammak Marwa, Yassine Boujelben, Noura Sellami, Iryna Andriyanova. Performance impact of packet multiplexing on massive multiplayer online games. Proceedings of the ACM Summer Computer Simulation Conference, Jul 2016, Montreal, Canada. hal-01709721

\section{HAL Id: hal-01709721 \\ https://hal.science/hal-01709721}

Submitted on 15 Feb 2018

HAL is a multi-disciplinary open access archive for the deposit and dissemination of scientific research documents, whether they are published or not. The documents may come from teaching and research institutions in France or abroad, or from public or private research centers.
L'archive ouverte pluridisciplinaire HAL, est destinée au dépôt et à la diffusion de documents scientifiques de niveau recherche, publiés ou non, émanant des établissements d'enseignement et de recherche français ou étrangers, des laboratoires publics ou privés. 


\title{
Performance impact of packet multiplexing on massive multiplayer online games
}

\author{
Marwa Dammak \\ LETI, ENIS \\ University of Sfax, Tunisia \\ dammakmarwa89@gmail.com
}

\author{
Yassine Boujelben \\ LETI, ENET'COM \\ University of Sfax, Tunisia \\ yassine.boujelben@enetcom.rnu.tn
}

\author{
Noura Sellami \\ LETI, ENIS \\ University of Sfax, Tunisia \\ noura.sellami@enis.tn
}

\author{
Iryna Andriyanova \\ ETIS, ENSEA \\ University Cergy Pontoise, \\ CNRS, France \\ iryna.andriyanova@ensea.fr
}

\begin{abstract}
Online games are more and more popular, resulting in an increasing share in the Internet traffic. These games generate a huge number of packets with typically small payloads, which overloads the network and deteriorates its efficiency. To mitigate these problems, it was proposed to use the tunnelling, compression and multiplexing (TCM) technique. Even though it was claimed via a set of emulations that this technique would enhance the network performance, there were no theoretical nor simulation studies to demonstrate its benefits on online gaming experience. In this paper, we implement TCM in NS-3 and set up online games scenarios in order to evaluate the performance of this technique in terms of delay, jitter and order of packets. The results show that with TCM the delay and jitter are more important than the native traffic for a small number of players due to the multiplexing periods. However, this situation is reversed when the number of players is high. Furthermore, the disorder of packets is always more important with TCM. In order to reduce the delay and the packets disorder, we propose a scenario where the online players are partitioned into subgroups based on their location on the game map. Simulations show that this leads to a significant performance improvement.
\end{abstract}

\section{Author Keywords}

performance evaluation; network delays; packet disorder, consistency, tunnelling, compression and multiplexing; NS3

\section{INTRODUCTION}

Massive Multiplayer Online Games (MMOG) are getting more and more popular all over the world. These games generate a great amount of traffic corresponding to an important share in the Internet resources. Indeed, online gaming traffic generated in 2014 had an average of 27 Petabytes per month [8].
This share is increasing year after year and will grow at a compound annual growth rate of $40 \%$ between 2014 and 2019 [8]. With the recent advent of cloud gaming and the gaming as a service (GaaS) solutions, the gaming traffic should become one of the largest Internet traffic categories [2].

The gaming traffic profile is composed of a huge number of small packets carrying event information produced by the online gamers. As events are generally position changes, these packets carry small payloads encapsulated within fixed length networking headers, which decreases the overall efficiency of the network. This is further aggravated when using TCP as transport protocol. Actually, the overall header size may represent in this case up to $73 \%$ of the online game traffic [5]. It becomes clear that finding a solution to this efficiency problem is an urgent necessity to deal with the increasing gaming traffic while maintaining an acceptable quality of experience for a kind of Internet users particularly demanding. Indeed, recent studies found that the probability of a player to quit the game is related to the quality of service (QoS) offered by the underlying network [6].

Online games, like any other real time applications, require a minimum network performance guarantee in terms of throughput and delay in order to offer an acceptable experience to the players. Several recent studies have been conducted in order to elaborate objective models to assess the quality of experience (QoE) in the context of online games. In [7], the authors have identified three key parameters to measure the QoE : responsiveness, precision and fairness. The responsiveness is related to the perception of the player to the overall game process. It measures the time taken by the system to undertake an event and display the corresponding changes on the player's screen. The precision represents the perception of the players to the results of their actions. It measures the closeness between the player's action and the system response. Finally, the fairness reflects the perception of players in comparison with each other. It represents the difference between all players' gaming environments. 


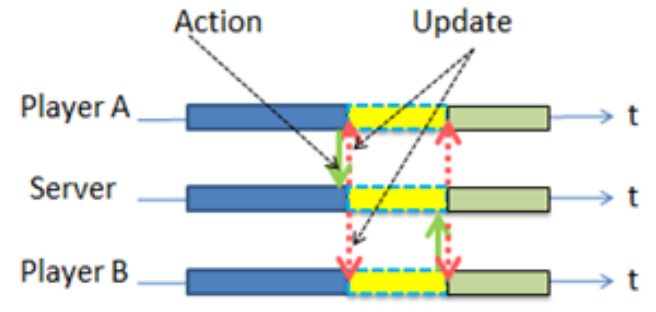

Consistency case

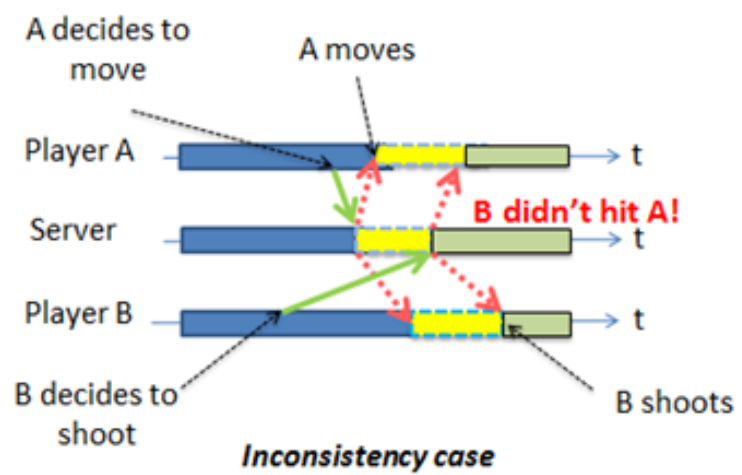

Figure 1. Consistency

Consistency has also been identified as a critical parameter for measuring QoE [7], [16]. The consistency of views reflects the coherence and synchronization of sequences observed by different players. A loss of consistency could make the game meaningless due to the loss of causality between the dependent events. Figure 1 shows an example where network transmission delay variation could entail serious causality problems, unless global reordering mechanisms are implemented. If the consistency is maintained as shown is the first case, then every action would be immediately updated to all players and would be the same view displayed for all of them. However, with network impairments, the consistency can't be maintained. The second case shows the effect of inconsistency on the game. In this scenario, player B shoots at player $\mathrm{A}$ at time $t_{1}$. Player $\mathrm{A}$ moves at time $t_{2}=t_{1}+\Delta$. However, the packet from player $\mathrm{B}$ has a higher transmission delay than the packet from player A. Thus, the action of A reaches the server sooner that the action of B. Hence, even though the action of B was taken before the action of A, player B couldn't hit the player A because it has already moved from the central server point of view.

These inconsistency problems are essentially caused by the delay, jitter and packet disorder impairments that the network introduces on the transmitted flows. Consequently, the network must provide acceptable QoS performance bounds in order to satisfy the increasing number of online gamers. While it is possible to enforce these bounds through complex end-to-end QoS architectures, we argue that proactive solutions should provide acceptable results with much less com- plexity. For example, in an underloaded network, congestion events should be minimized and therefore the data flows are very unlikely to experience excessive delays or packet loss impairments. In this work, we will evaluate the performance of two proactive techniques that aim to reduce the network load. The first technique is Tunnelling, Compression and Multiplexing (TCM) and the second is multicast group partition.

The main contributions of this paper are as follows. First, we develop on top of the network simulator NS3 a simulation framework for studying the network gaming performance with realistic inputs from gaming traces. Second, we set up tree-based and forest-based simulations and evaluate the performance of TCM and group partition in terms of delay, jitter and packet order. We choose a first person shooter game called Quake III to build the traffic model for these simulations.

The remainder of this paper is structured as follows. Section II summarizes the related works. Section III describes the TCM technique and discusses the limitations of its current implementation. Section IV presents our proposition to enhance the TCM algorithm performance and a description of the various configurations that will be simulated. In section V, we discuss the simulation results. Finally, section VI concludes this work.

\section{RELATED WORKS}

Massive multiplayer online game (MMOG) is a group communication service that allows thousands of players to simultaneously interact with each other via the Internet. A typical implementation of an MMOG infrastructure is based on a multicasting model and a client-server architecture. The main reason for using multicasting is to reduce the amount of traffic in the downstream direction, while using a centralized client-server architecture allows better control of consistency. Scalability is often achieved by using game proxies as shown in figure 2. When a player performs an action, a message is sent to its corresponding game proxy which might queue it for possible multiplexing and then forward the whole gathered actions to the central server. Periodically, the central server sends an update message to all players to inform them of the new global situation through the multicast tree. It is important to note that the multicasting topology is not necessarily implemented in the network layer, application-level multicast (ALM) is generally used for MMOG in order to get around the multiple obstacles facing the native multicast [3]. In this context, we note also the use of the peer-to-peer overlays [10]. Besides tree-based, ring-based topologies have also been proposed for multiplayer games. In [1], the authors proposed a multiring topology for connecting subgroups of players within the same game in order to enforce consistency in a more distributed way.

In addition to these researches on the MMOG infrastructures, many studies have been established in order to characterize the gaming traffic and to propose some solutions to overcome the network efficiency decrease. They have shown that this traffic is basically composed of very tiny packets [4] [19] with a small payload size. Actually, the traffic is mostly composed 


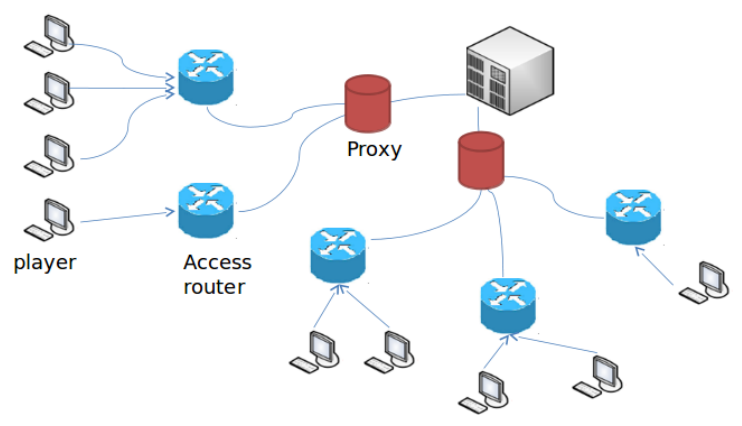

Figure 2. Typical online game infrastructure

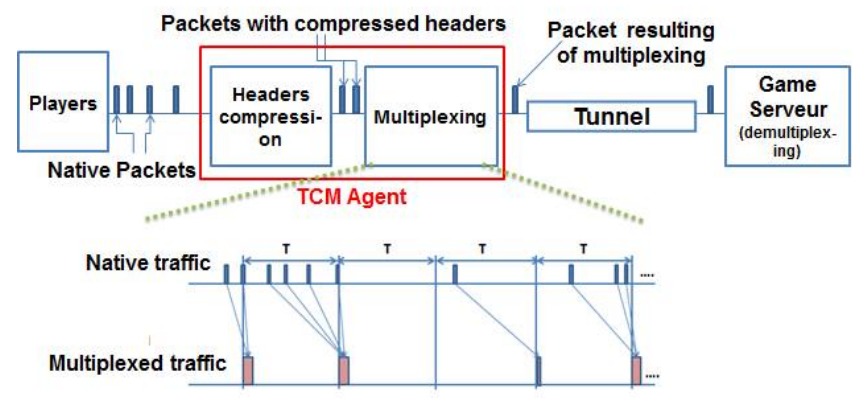

Figure 3. TCM steps

of headers especially if the transport protocol used is TCP where the headers and the acknowledgements can represent up to $73 \%$ of the traffic [5]. This traffic is also characterized by its periodicity which causes the flash crowds effect since the network will receive a huge number of packets at almost the same time. These bursts impose some challenges to the network infrastructure in terms of processing, buffering and delays [19]. Obviously, The flash crowd effects become increasingly challenging as the number of players gets larger.

It is therefore clear from the above discussions that by reducing the overall amount of gaming traffic overhead and the number of transmitted packets, it will be possible to make significant performance enhancement. In this context, TCM is a very interesting candidate since it allows traffic compression as will be explained in the next section.

\section{TUNNELLING, COMPRESSION AND MULTIPLEXING}

TCM (tunnelling, compression and multiplexing) is a method introduced in [9] as a solution to minimize the internet traffic charge. It was proposed in [17] in order to reduce the overhead of online games traffic. Figure 3 describes TCM steps.

The packets are received by the TCM agent which can be located at the access provider proxies or directly at the clients as a software. The TCM agent will then assure the header compression, the multiplexing and the tunnelling. In [17] and [18], TCM has been proposed as a solution to reduce the traffic charge of online games. However, there were no simulations to confirm the benefits of TCM. The gain has been demonstrated using an emulation based on fixed values of delay. In this paper, we will setup different simulations to

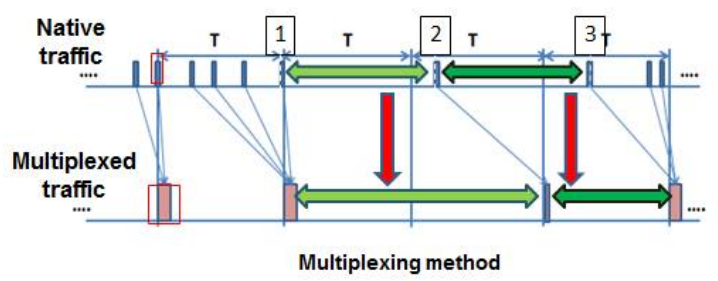

Figure 4. TCM jitter

evaluate the effect of applying TCM. Moreover, we will simulate the forest topology and evaluate its performance with and without TCM. In the following, we describe these steps in more details.

Header Compression When a packet is received by the TCM agent, the UDP/IP or TCP/IP header will be compressed using a specified header compression protocol. The protocols that can be used for online games are IPHC and ROHCv2 [18]. This compression can reduce the IP header from 20 to 2 bytes and the UDP or TCP header from 8 or 20 bytes, respectively, to 2 bytes as well.

Multiplexing After the header compression, the packets will be multiplexed and merged into one packet. The multiplexing technique proposed in [17] and [18] for online games is based on a period of time $T$ : the agent will receive the packets, compress their headers and wait until the period expires to multiplex them. In case the agent receive juste one packet, nothing will be done and the packet will be sent as it is since multiplexing will increse its size.

Tunnelling The last step is to send the multiplexed packets to the server using a layer 2 Tunnelling Protocol. Once received, the packets will be demultiplexed and restored to their original form.

The main limitations of TCM can be summarized into three problems. First, an additional delay will be incurred by native packets since they need to wait until the end of the multiplexing period $T$ to be sent. This delay is proportional to the period and is added to all the packets with an average of $T / 2$. Second, since this additional delay changes from one packet to another, depending on the time the packet is received by the TCM agent, a variable jitter delay should also be added as explained in figure 4 . If we consider native packets 1 , 2 and 3, we can see that due to the TCM period, the delay between consecutive multiplexed packets carrying 1 and 2 has remarkably increased, while delay between multiplexed packets carrying 2 and 3 has decreased. Third, if the number of native packets gathered within a single period is high, the multiplexed packet size will be very high as well, which may require using segmentation in the lower layers.

In the next section, we will propose a new approach for implementing the TCM algorithm that should address the third limitation. For the first two ones, we will use simulations to evaluate the net gain of delay and when is it worth using TCM for MMOG.

\section{SYSTEM DESCRIPTION}


In order to accelerate software downloads and improve user experience for all gamers regardless of their geographic locations, game providers are more and more using CDN (content delivery networks) services, e.g., Akamai game delivery network. According to this new trend, the game network is typically made of a central server and a set of proxy servers which connect the access routers and finally the end users as shown in figure 2 .

The interactions between the users and the servers are based on a point-to-multipoint model in the downlink direction and a multipoint-to-point model in the uplink direction. The traffic is therefore unicast in the uplink and multicast in the downlink directions. These network entities are typically connected via a tree-based topology.

The online game we choose for the simulations is Quacke III. The traffic in both directions is described as follows.

- Unicast : an approximately periodic traffic with a period around $10.75 \mathrm{~ms}$ [11]. The packet size has a mean of 70 bytes per client.

- Multicast : a periodic traffic with a period around $50 \mathrm{~ms}$ [11]. Every period the server sends an update message with a size that depends on the number of players in the game. The size of the packet can be calculated using the approximate formula proposed in [11]:

$$
\text { Size }_{\text {Server }}=81.9+13 *\left(N_{\text {Players }}-2\right)
$$

where Size $e_{\text {Server }}$ refers to the size of the server's packet and $N_{\text {Players }}$ is the number of players connected to the server.

In order to avoid multiplexed packet segmentation, we introduce a new procedure to TCM that allows multiplexing and sending a packet by the TCM agent after receiving $N_{M a x}$ packets from the players even before reaching the end of the period. In this case, we could limit the maximum size of the multiplexed packet and by the way reduce the additive delay to native packets. The new TCM routing algorithm is described with further details in the following pseudo-code.

\subsection{Group partition}

In order to minimize the traffic charge and the delay, we propose to divide the players into subgroups. This idea was adopted in many other works where the multicast group is partitioned according to a specific criteria. As an example, in [12] [14] the criteria chosen was the receiver's interest and, in [13] it was the packet loss correlation. Another criteria was used in [15] which is the data encoding method. In our case, we can divide the players into subgroups based on their location in the game's map. Indeed, a player needs to be aware of the instant action of only players in his neighbourhood. Certainly he also needs to have an update about all players' movements, but these updates called global updates can be sent less often than the local updates (updates about members of the same subgroup). This subdivision allows to have a certain independence among the groups in terms of traffic transmission. Therefore, the new topology will be called a forest topology. To explain this idea of partition, we will refer to figure 5 and figure 6.
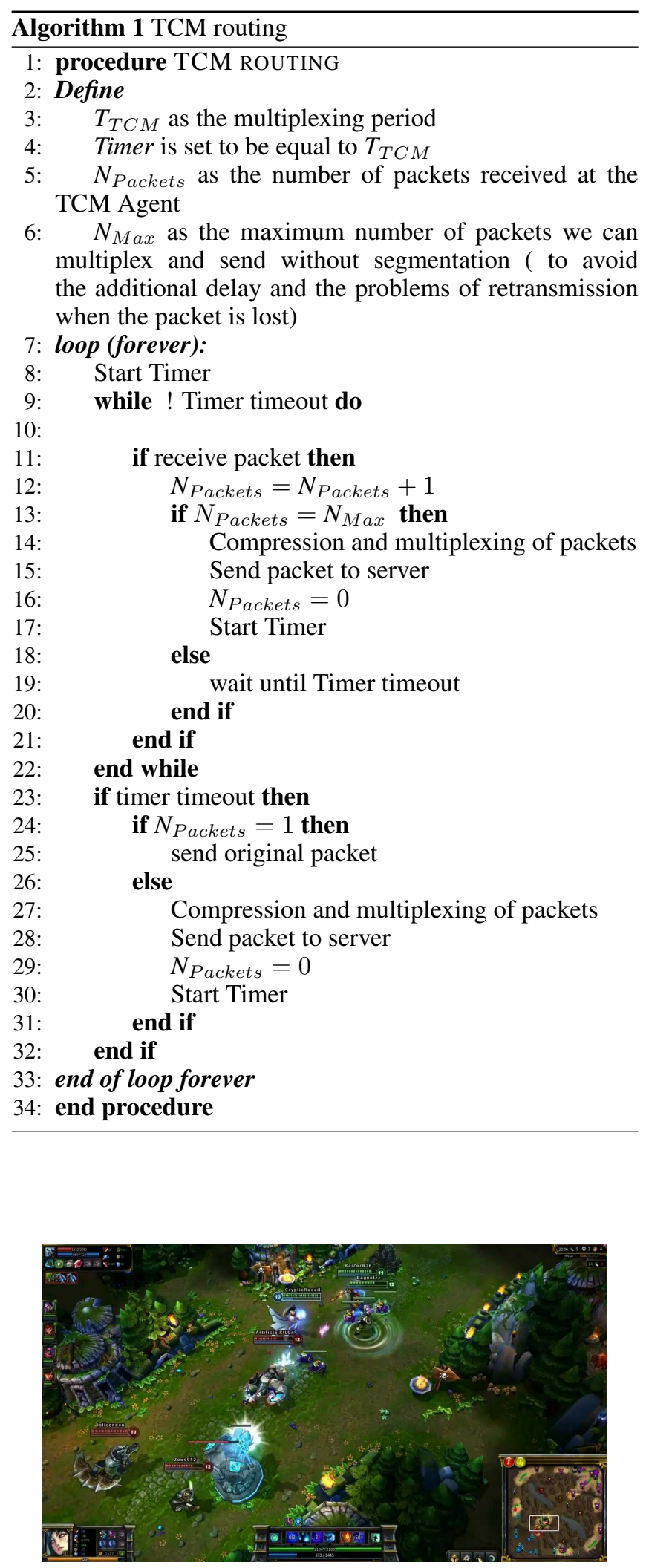

Figure 5. League of Legends players screen 


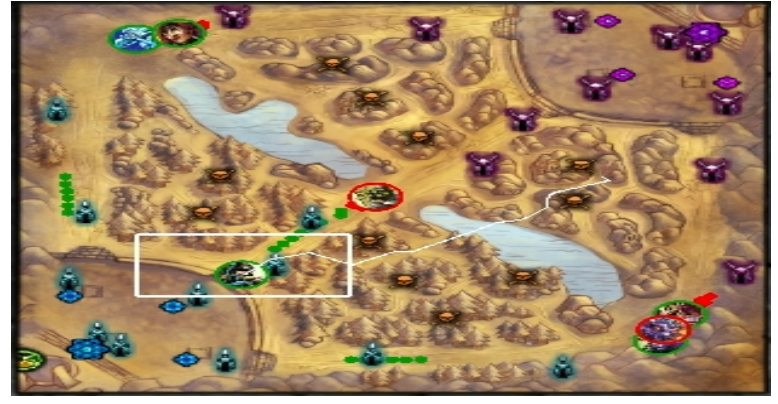

Figure 6. League of Legends global vision

These figures are two screen shots from an active session of a known MMORPG game called League Of Legends. In this game, the players are divided into two teams. Each team has a number of nexus that need to be protected. These nexus is actually the primary objective of the game. In order to win the match, a team has to destroy the opposing team's nexus. Each player with his team members will attack the other team's nexus. Therefore, they will be divided into groups and try to destroy the nexus by attacking the towers protecting it which are placed far away from one another. Hence, the player will be focused on the battle he is having and won't see what is exactly going on with the other players in other places on the map as we can see in figure 5. He can have a global vision of the game which gives an idea about the approximate position of all players and the state of the towers and nexus as shown in figure 6. Based on this scenario, we can divide the players into subgroups based on their position on the map (for example around each tower). For each subgroup we define a local server, which will be responsible for receiving the subgroup players actions and multicasting the periodic updates to them. It will send another update about its subgroup to the central server but less frequently. After receiving the updates, the central server will send a global update about all the players to all subgroups. This subdivision will help to minimize the traffic destined for the central server of the game and the delay at the same time. In the following, we explain with more details this proposed topology.

\subsection{Forest}

According to graph theory, a forest is a collection of trees not necessarily interconnected. In this work, we will only use interconnected subtrees which represent a tree from a topological point of view. However, since the traffic in each subtree may be different from another, we will call this topology a forest with respect to this traffic independence. Each subtree connects a subgroup of players chosen based on their location in the game's map (neighbouring players). The forest is composed of multiple subtrees having the proxy servers as roots. These subtrees are interconnected to the central server which represents the root of the forest as shown in figure 7.

For the forest topology we have four types of traffic.

- Local Unicast : from players of a subgroup to the local server of that subgroup. This traffic is similar to unicast traffic for the tree.

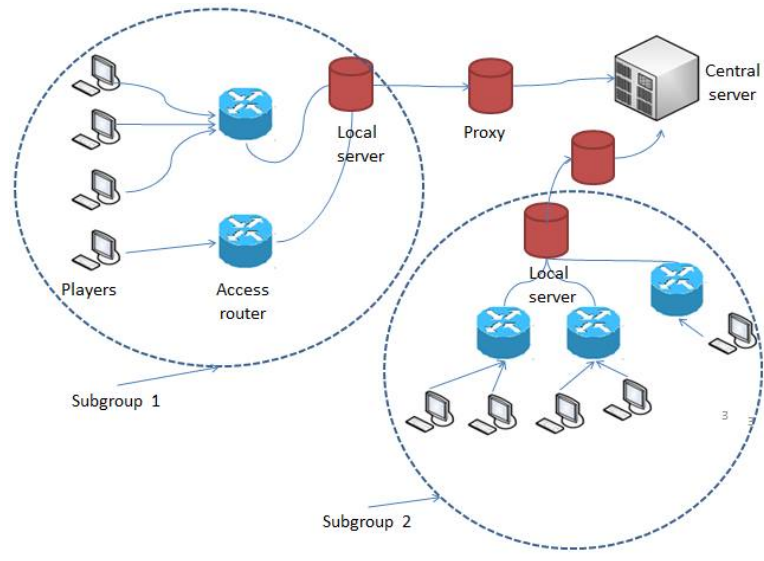

Figure 7. Forest Topology

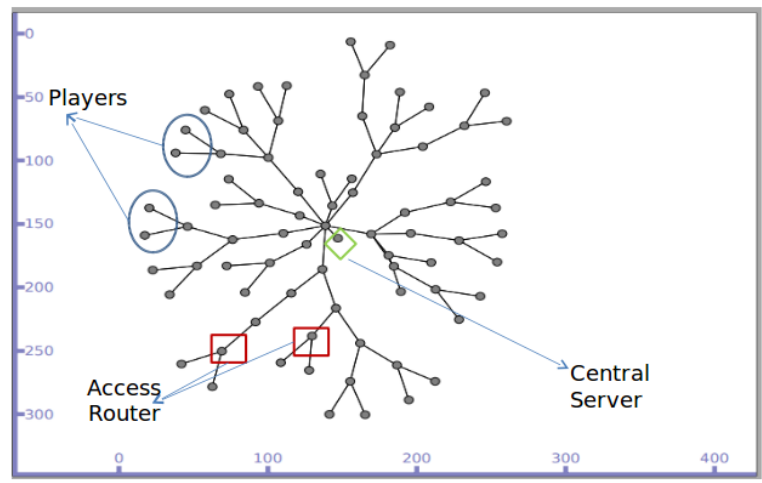

Figure 8. Tree Topology by NS3

- Local Multicast : from the local server of a subgroup to the players of the subgroup. It has the same characteristics of multicast traffic in the tree topology.

- Global Unicast : from the local servers to the central server. In order to conserve the $80 / 20$ rate relation between the local and the global traffic, we set up the local server to send an update packet about his subgroup after receiving four players packets. The size is calculated with the same formula as the local multicast traffic.

- Global Multicast : from the central server to all the players. When it receives a global unicast packet, the server will generate an update packet, which size is also calculated the same way as the local multicast packets.

\section{SIMULATIONS AND RESULTS}

In order to evaluate the impact of applying TCM for the online games and to measure the effect of dividing the players into subgroups as explained previously, we set up four simulation scenarios: Tree without TCM, Tree with TCM, Forest without TCM and Forest with TCM. For each input value, we run 10 simulations and take the mean of their results.

To realize these simulations, we use the NS3 simulator. Indeed, the graphs are generated randomly using GT-Itm graph generator. Then, we use the Dijkstra algorithm in order to obtain the tree and the forest topologies based on the shortest 


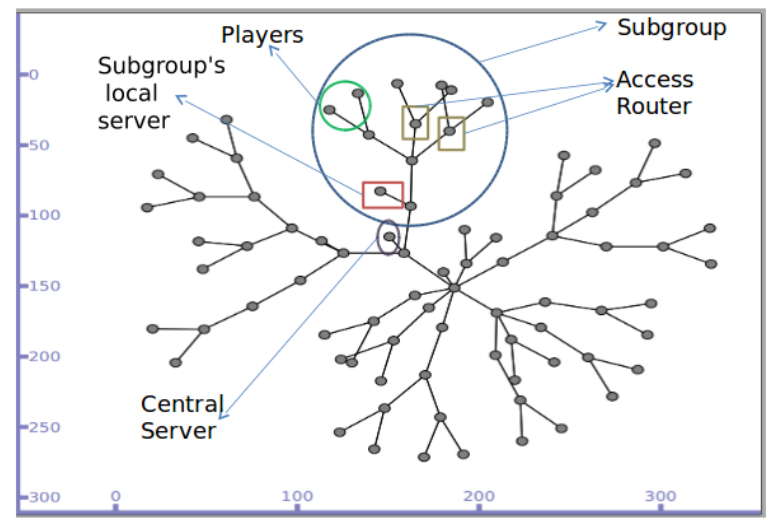

Figure 9. Forest Topology by NS3

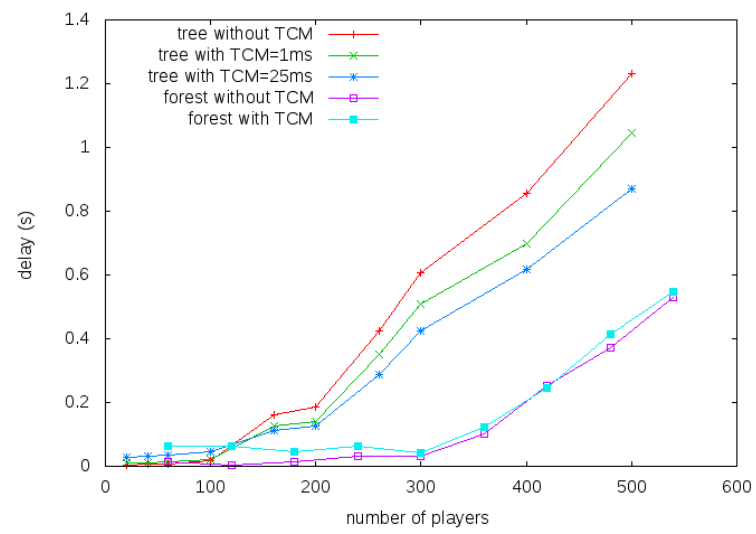

Figure 10. TCM allows better delay performance at heavier loads

path calculation. Finally, we calculate their adjacency matrix to generate them with NS3. The resulting topologies are represented in figure 8 and figure 9 .

We add an FTP client to generate a Poisson background traffic representing 50\% of links capacities. The capacities of links are set as follows. Access links are set to $1 \mathrm{Mbps}$, Metro links to $5 \mathrm{Mbps}$ and Core links to $10 \mathrm{Mbps}$. These values are chosen in order to reach the network congestion when the number of players increases in the simulations. The players send their actions periodically with a slight random delay representing the difference between their different processors.

\subsection{Delay}

The first parameter we are studying is the impact of using TCM, for both tree and forest topologies, on the end-to-end delay as the number of players and therefore the traffic load increases. Figure 10 summarizes the results found with the simulations.

When using tree topologies, the delay with TCM is greater than the delay for native traffic when there are only few players in the game. Actually, in this case the gain in bandwidth with TCM is not very important. Besides, a delay caused by the multiplexing period $T$ will be added. When the number of players increases, the delay increases exponentially, but in this case it becomes greater with the native traffic than

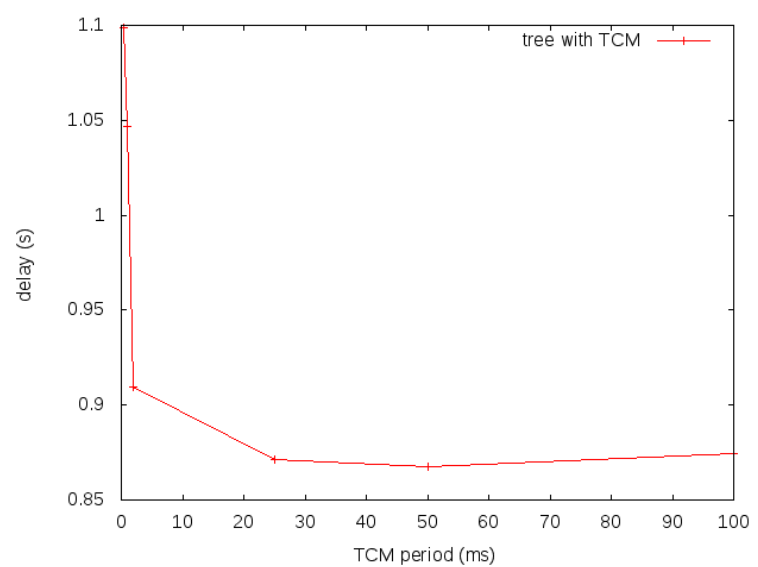

Figure 11. Delay performance is better for larger TCM periods

with TCM. Indeed, TCM allows better performance at heavier load. We note that the delay difference tends to increase with the number of players. We have also highlighted the impact of the multiplexing period. We note that the delays decrease for larger periods. Obviously, this may not be a general trend, this is why, we will make a specific study according to th multiplexing period to asses the exact behaviour.

When using forest topologies, the simulation results show that the end-to-end delay is on average $400 \mathrm{~ms}$ lower than with the tree topology even with the TCM method. Moreover, we note the advantage of using partition on the overall network load. Actually, for the same number of players, there wasn't a difference between using native traffic or applying TCM routing because we have reduced the offered traffic load, especially on bottleneck links.

Finally, we make a specific study to assess the impact of the multiplexing period on the delay performance. We fixe the number of players to 500 and we increased the value of TCM period from 0 to $100 \mathrm{~ms}$. Figure 11 shows the delay with respect to the TCM period for a tree topology. The results show, as expected, that when the period of multiplexing increases, the delay decreases since the bandwidth used is reduced. However, we can't increase this period infinitely. Indeed, as shown in the figure, at some point the delay becomes almost the same no matter how much we increase the period. This result is expected as it is shown in [17]. Actually, when the period or the number of players is high, the gain in bandwidth will meet an asymptotic limit given by the following expression :

$$
B W R_{a}=\frac{M H+\mathbb{E}[R H]+\mathbb{E}[P]}{N H+\mathbb{E}[P]}
$$

where $B W R$ is the bandwidth relationship calculated by dividing the bandwidths with and without multiplexing, $M H$ denotes PPPMux header included at the beginning of each compressed packet, $R H$ the reduced header, $N H$ the UDP/IP header and $P$ is the payload of the native packets.

We also note that we obtain a slight increase for very high multiplexing periods as we delay packets further. Therefore, 


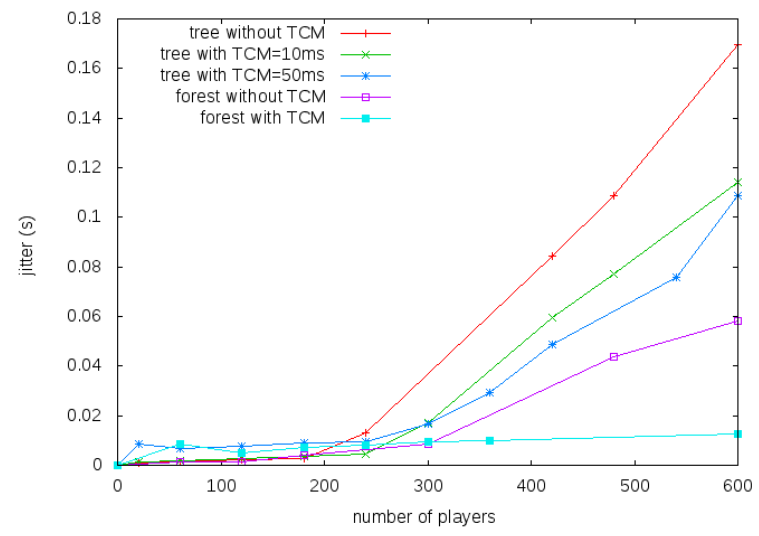

Figure 12. Jitter variation with the number of players

we can state that there is no interest in delaying packets further than $T=50 \mathrm{~ms}$.

\subsection{Jitter}

The second parameter influencing the QoE of online games is the jitter. First, we compute the variation of this jitter with the number of players connecting to the game. The results of our simulation are shown in figure 12

When using tree topologies, TCM traffic shows higher jitter for a small number of players, which is due to the TCM multiplexing period as explained previously. The results change when the number of players increases. For our set up, when the number of players is more than 200 , the jitter of native traffic becomes more important. Indeed, when we have more players, we have more successive packets so that the average jitter caused by the TCM period decreases. Moreover, since the traffic is reduced with TCM, the jitter becomes smaller than with the native traffic. As for the tree with TCM periods, when we have a few number of players, the bigger the period is , the higher the jitter generated is. This case changes when the number of players is beyond 300 as the number of multiplexed packets becomes higher with a bigger period. The jitter also shows an asymptotic limit.

When using forest topologies, the jitter values obtained are even better than those of the tree with TCM. When we apply TCM for the forest, the jitter becomes very low which will offer a better user experience for online games players.

Finally, we make a new set of simulations to evaluate the impact of the multiplexing period on the jitter for 600 players. As shown in figure 13, the jitter is proportional to the TCM period : a bigger period results in a higher jitter. The jitter also follows an asymptotic limit for a big number of players and high periods.

\subsection{Order}

Since consistency is proven to be a critical requirement for network gamers, we are interested in evaluating the number of out-of-order packets delivered by the network to the gaming applications. We believe that inconsistency is mainly due to packets displayed in the wrong order.

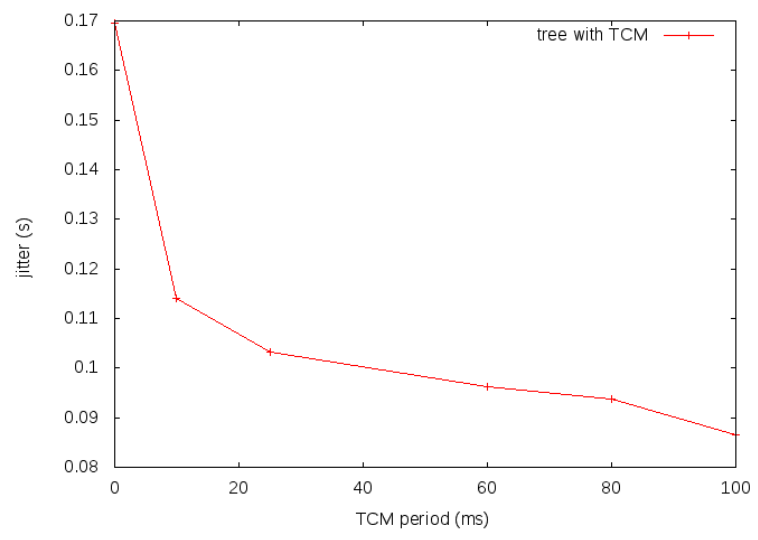

Figure 13. Jitter in function of TCM period

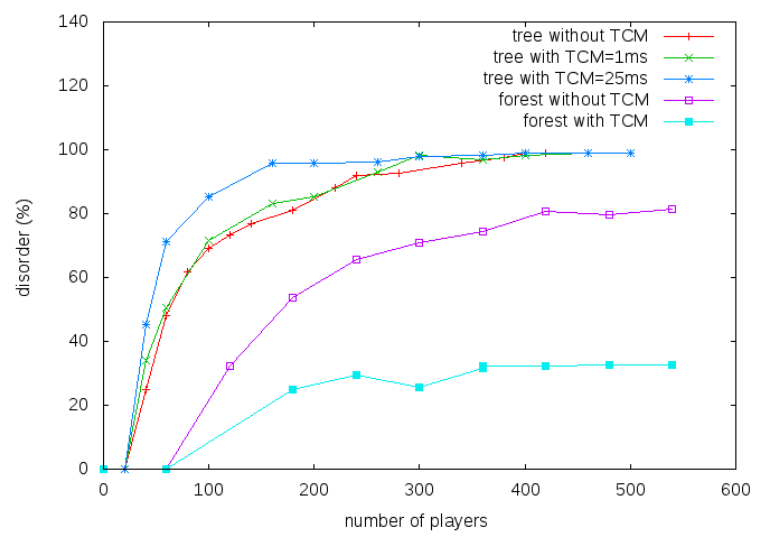

Figure 14. Number of out-of-order packets as a function of the number of players

First, we compute the number of packets delivered out-oforder as the number of players increases for tree and forest topology.

Figure 14 shows that the number of out-of-order packets increases with the number of players for tree topology. Even though with TCM the traffic is reduced, we find that the number of out-of-order packets is always greater than with native traffic. This result can be explained by the fact that with TCM, if a packet is not received in order, all the native packets multiplexed in that packet will not be received in order as well, which leads to a burst of out-of-order packets.

For forest topologies, the results in figure 14 also points out that the group partition method can considerably decrease the out-of-order packets rate. It is even less when we add TCM. This difference between the tree and the forest topologies can be explained by the network load decrease.

Second, we fixe the number of players to 200 and calculate the out-of-order rate for different TCM periods.

Figure 15 shows that the disorder increases as the TCM period increases. Actually, with TCM, the disorder is important since one out-of-order packet implies the disorder of all the multiplexed packets. Moreover, with a larger period, more packets are multiplexed at once. Thus, the disorder increases 


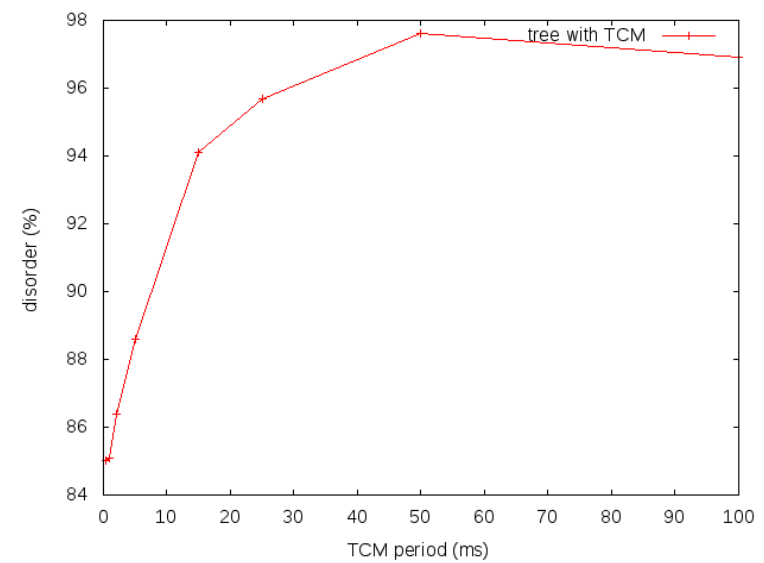

Figure 15. Variation of the number of out-of-order packets with the TCM period

when the period of TCM increases and could reach a case where almost all the packets are out-of-order, which would require an extensive effort of reordering, and therefore, additive processing delay.

\section{CONCLUSION}

In this paper, we were interested in assessing the effect of packet multiplexing on the QoE of online multiplayer games. Therefore, we studied the three major parameters influencing this QoE : the delay, the jitter and the percentage of out-oforder packets delivered to the applications. The simulations showed that TCM is better than the simple routing in terms of delay and jitter when the number of players increases. However, in terms of disorder, the simple routing showed better results than TCM. We also studied the case where we divide the players into subgroups based on their location in the game's map to form the "forest". This method can decrease the delay and the disorder and hence enhance the QoE of online games.

\section{REFERENCES}

1. Boujelben, Y., Girard, A., and Grgoire, J.-C. A sequential algorithm for constructing delay-constrained multirings for multipoint-to-multipoint communications. Telecommunication Systems (2006), 43-59.

2. Cai, W., Chen, M., and Leung, V. C. Toward gaming as a service. IEEE Internet Computing 18 (2014), 12-18.

3. Castro, M., Druschel, P., Kermarrec, A.-M., and Rowstron, A. Scribe : A large-scale and decentralized application-level multicast infrastructure. IEEE journal on selected areas in communications 20 (October 2002).

4. Chen, K.-T., Huang, P., Huang, C.-Y., and Lei, C.-L. Game traffic analysis: An mmorpg perspective. NOSSDAV05, Stevenson, Washington, USA (June 2005).

5. Chen, K.-T., Huang, P., and Lei, C.-L. Game traffic analysis: An mmorpg perspective. Computer Networks (2005), 3002-3023.

6. Chen, K.-T., Huang, P., and Lei, C.-L. Effect of network quality on player departure behavior in online games.
Parallel and Distributed Systems, IEEE Transactions (may 2009), 593-606.

7. Chen, P., and Zarki, M. E. Perceptual view inconsistency: An objective evaluation framework for online game quality of experience (qoe). Network and Systems Support for Games (NetGames) (Octobre 2011).

8. Cisco. Cisco visual networking index: Forecast and methodology, 2014-2019 white paper. Information Technology: New Generations (ITNG),Tenth International Conference (May 2015).

9. J.Saldana, Wing, D., Fernndez-Navajas, J., Ruiz-Mas, J., Perumal, M. A. M., and Camarillo, G. Widening the scope of a standard: Real time flows tunneling, compressing and multiplexing. IEEE International Conference on Communications (ICC) (June 2012), $6906-6910$.

10. Knutsson, B. ., Lu, H., Xu, W., and Hopkins, B. Peer-to-peer support for massively multiplayer games. IEEE INFOCOM (2004).

11. Lang, T., Branch, P., and Armitag, G. A synthetic traffic model for quake3. ACE04, Singapore (June 2004).

12. Levine, B., Crowcroft, J., Diot, C., Garcia-Luna-Aceves, J., and Kurose, J. Consideration of receiver interest for ip multicast delivery. Proc. IEEE INFOCOM2000 (2000), 470-479.

13. Levine, B., Paul, S., and Garcia-Luna-Aceves, J. Organizing multicast receivers deterministically according to packetloss correlation. Proc. Sixth ACM International Multimedia Conference (September 1998).

14. Macedonia, M., Zyda, M., Pratt, D., Brutzman, D., and Barham, P. Exploiting reality with multicast groups. IEEE Computer Graphics and Applications (1995).

15. McCanne, S., Jacobson, V., and Vetterli, M. Receiver-driven layered multicast. ACM SIGCOMM (1996), 117-130.

16. Palant, W., Griwodz, C., and Halvorsen, P. Consistency requirements in multiplayer online games. NetGames '06 Proceedings of 5th ACM SIGCOMM workshop on Network and system support for games, USA (October 2006), 1-6.

17. Saldana, J., Fernandez-Navajas, J., Ruiz-Mas, J., and Casadesus, L. Online games traffic multiplexing: Analysis and effect in access networks. KSII Transactions on Internet and Information Systems (November 2012), 2920-2939.

18. Saldana, J., Murillo, J., Fernndez-Navajas, J., Ruiz-Mas, J., Aznar, J. I., and Navarro, E. V. Bandwidth efficiency improvement of online games by the use of tunneling, compressing and multiplexing techniques. Proc. International Symposium on Performance Evaluation of Computer and Telecommunication Systems SPECTS (2011), 227-234.

19. W.C.Feng, F.Chang, W., and J.Walpole. A traffic characterization of popular on-line games. IEEE/ACM Transactions on Networking, (June 2005). 